\title{
Doença do Espectro Neuromielite Óptica (DENMO)
}

\author{
Neuromyelitis Optica Spectrum Disorders (NMOSD)
}

\author{
Barbara Kelly Gonçalves Azevedo ${ }^{1}$, Monica Seixas Oliveira ${ }^{1}$, Valter Alves de Moura \\ Neto $^{1}$, Jamile Seixas Fukuda ${ }^{1}$, Thiago Gonçalves Fukuda ${ }^{2}$ \\ ${ }^{1}$ Residente do Serviço de Neurologia do Hospital Santa Izabel; ${ }^{2}$ Serviço de Neurologia do \\ Hospital Santa Izabel; Salvador, Bahia, Brasil
}

\begin{abstract}
Correspondence addresses:
Dr. Thiago Gonçalves Fukuda thiagofukuda@gmail.com
\end{abstract}

Received: April 9, 2019

Revised: May 31, 2019

Accepted: June 12, 2019

Published: June 28, 2019

Data Availability Statement: All relevant data are within the paper and its Supporting Information files.

Funding: This work was the result of authors' initiative. There was no support of research or publication funds.

Competing interests: The authors have declared that no competing interests exist.

Copyright

(C) 2019 by Santa Casa

de Misericórdia da Bahia.

All rights reserved.

ISSN: 2526-5563
As Doenças do Espectro Neuromielite Óptica (DENMO) são doenças imunomediadas do sistema nervoso central (SNC) associadas à presença de um marcador biológico de alta especificidade, o anticorpo anti-aquaporina-4. É uma doença rara com prevalência mundial em torno de $0,5-1,0$ por mil habitantes, mais frequente no sexo feminino com uma relação de 10 mulheres para cada homem acometido e mais incidente em indivíduos de descendência africana e asiática. As manifestações clínicas mais características são a neurite óptica grave, a mielite transversa longitudinal extensa e a síndrome de área postrema do bulbo (caracterizada por náuseas, vômitos e soluços recorrentes). Os sintomas apresentam-se em forma de exacerbações agudas e períodos de remissão. $O$ diagnóstico é baseado nos critérios do Painel Internacional para Diagnóstico NMO, que definiu os critérios diagnósticos atuais em 2015 a partir dos sintomas clínicos típicos e na positividade ou não do anticorpo anti-aquaportina-4. $O$ tratamento das exacerbações agudas é realizado com corticoterapia endovenosa de 3-5 dias associado ao uso de plamaférese em casos graves ou refratários ao tratamento com corticoide. $O$ tratamento de prevenção das exacerbações agudas utilizado atualmente é baseado em trabalhos de baixo poder científico, considerando como primeira linha de tratamento imunossupressores como azatioprina, micofenolatomofetila e o rituximabe. Entretanto, novos medicamentos com alvos terapêuticos específicos foram estudados nos últimos anos com boa perspectiva para o tratamento futuro da DENMO.

Palavras-chave: DENMO; Neurite Óptica; Mielite; Aquaporina-4.

Neuromyelitis Optica Spectrum Disorders (NMOSD) are immune-mediated diseases of the central nervous system associated with the presence of a high specificity biological marker, the anti-aquaporin- 4 antibody. It is a rare disease with a world prevalence of around $0.5-1.0$ per thousand inhabitants, more frequent in females with a ratio of 10 females for each affected man and more incident in individuals of African and Asian descent. The most characteristic clinical manifestations are severe optic neuritis, extensive longitudinal, transverse myelitis and area postrema syndrome (characterized by recurrent nausea, vomiting, and hiccups). Symptoms present as acute exacerbations and periods of remission. The diagnosis is based on the criteria of the International Panel for NMO Diagnosis that defined the current diagnostic criteria in 2015 based on the typical clinical symptoms and the anti-aquaporin-4 antibody positivity or non-positivity. The treatment of acute exacerbations is generally with 3-5 days intravenous corticosteroid therapy associated with the use of plasmaphereses in severe cases or refractory to treatment with corticosteroids. The prevention treatment of acute exacerbations currently used are based on works of low scientific power, considering as the first line of treatment immunosuppressants like azathioprine, mycophenolate, and rituximab. However, new drugs with specific therapeutic targets have been studied in recent years as a good prospect for the future treatment of NMOSD.

Keywords: NMOSD; Optic Neuritis; Myelitis; Aquaporin-4. 


\section{Introdução}

Neuromielite óptica (NMO) e as doenças a ela relacionadas (espectro neuromielite óptica - DENMO) são desordens imunomediadas, inflamatórias e desmielinizantes do SNC. ${ }^{1,2}$

Até 2004, a NMO e DENMO eram consideradas variantes da esclerose múltipla (EM). Neste ano, a descoberta de um marcador, um autoanticorpo sérico altamente específico para NMO (NMO-IgG), possibilitou a revisão desses conceitos, corroborando para uma maior compreensão das duas entidades. ${ }^{3}$ Atualmente, achados clínicos, imunológicos, patológicos e radiológicos distinguem a NMO e DENMO da esclerose múltipla clássica. ${ }^{4,5}$

\section{Epidemiologia}

A prevalência de NMO é bastante variável nos estudos atuais, entre $0,5-1,0$ por 100.000 habitantes. ${ }^{6}$ É provável que esta prevalência se eleve com o aumento da sensibilidade e disponibilidade dos testes AQP4-IgG. A média de idade na apresentação é de 39 anos, com casos também descritos em crianças e na população idosa. $^{7,8}$

As mulheres são mais comumente afetadas, com incidência até 10 vezes maior quando comparada aos homens. ${ }^{7,9}$ Casos de NMO foram relatados em todos os continentes e raças. $\mathrm{Na}$ Europa, a prevalência de NMO entre os distúrbios autoimunes do SNC é menor em comparação com a esclerose múltipla. No entanto, a NMO representa uma proporção substancial de desordens inflamatórias desmielinizantes do SNC em populações não caucasianas, como afro-brasileiros (15\%), leste-asiáticos (até 48\%) e indianos $(9 \%)$. $^{1,7,8}$

A NMO é geralmente esporádica, embora alguns casos familiares tenham sido relatados. ${ }^{10}$ Foi observada uma associação entre o distúrbio e outras doenças autoimunes como a miastenia gravis, lúpus eritematoso sistêmico, síndrome de Sjögren, doença do tecido conjuntivo e artrite reumatoide. Há ainda relato de coexistência clínica rara entre DENMO e encefalite antireceptor N-metil-D-aspartato (NMDA), podendo a encefalite preceder, acompanhar ou ter apresentação posterior a episódios de DEMNO. ${ }^{8,13}$

\section{Patogênese}

O anticorpo NMO é um autoanticorpo IgG que se liga especificamente à AQP4, uma molécula de proteína que forma um canal transmembrana e facilita o transporte de água através das membranas celulares em resposta a gradientes osmóticos. Expressa-se principalmente nos astrócitos presentes no nervo óptico, na medula espinhal, nas áreas periventriculares, hipotálamo, tronco encefálico e na área postrema. ${ }^{14}$

No início da formação da lesão, AQP4-IgG liga-se à AQP4, ativa o complemento e provoca deposição de complexos de ataque à membrana. As citocinas recrutam neutrófilos e eosinófilos em espaços perivasculares. Os neutrófilos, por sua vez, degranulam para causar a morte de astrócitos que leva à morte de oligodendrócitos, ocorrendo a morte neuronal. Lesões são caracterizadas por necrose completa do tecido e infiltração generalizada por macrófagos. ${ }^{7}$

A AQP4 também pode estar presente fora do $\mathrm{SNC}$, encontrada nos rins, células parietais gástricas, vias aéreas, músculos esqueléticos e glândulas. Entretanto, até o momento, as alterações patológicas da NMOSD estão limitadas ao SNC. ${ }^{14}$

Uma menor parcela de pacientes soronegativos para AQP4 com fenótipo dos distúrbios possui anticorpos séricos contra a glicoproteína oligodendrocítica de mielina (MOG), podendo definir uma síndrome clínica sobreposta ou distinta. ${ }^{15,16}$

As principais características clínicas da NMO incluem ataques agudos de neurite óptica bilateral, ou rapidamente sequencial ou mielite transversa com curso redicivante. ${ }^{1,2,7}$

A neurite óptica apresenta graus variados de perda de visual, normalmente associada à dor ocular e à mobilização. A perda visual é mais 
grave na NMO quando comparada à esclerose múltipla. ${ }^{17}$

A mielite transversa é uma disfunção que se desenvolve ao longo de horas ou dias, na ausência de lesão estrutural medular e, em geral, envolve três ou mais segmentos vertebrais, que podem ser vistos na ressonância magnética, quando então é denominada mielite transversa longitudinalmente extensa (LETM). Na NMO, a desmielinização é mais grave quando comparada à desmielinização na esclerose múltipla. ${ }^{14,17}$

Outros sintomas sugestivos de NMO incluem episódios de náuseas e vômitos intratáveis, soluços, sonolência diurna ou narcolepsia, obesidade, distúrbios neuroendócrinos, que reforçam o comprometimento do SNC, além da medula espinhal e nervos ópticos. ${ }^{2}$

A NMO tem um curso recidivante em $80 \%$ $90 \%$ ou mais dos casos. A recaída ocorre no primeiro ano após um evento inicial em $60 \%$ dos pacientes e em três anos em $90 \%{ }^{17}$

Pacientes soropositivos para AQP4-IgG geralmente apresentam ataques clínicos mais graves, pior prognóstico, mais recaídas (81\%-91\%) quando comparados a pacientes soronegativos para AQP4-IgG. ${ }^{14}$

\section{Critérios Diagnósticos}

O Painel Internacional para Diagnóstico NMO (IPND) definiu os critérios diagnósticos atuais em 2015 (Tabela 1), estratificando os pacientes de acordo com o status AQP4-IgG, caracterísicas clínicas e achados em neuroimagem. ${ }^{18}$ Os critérios de 2015 melhoraram acentuadamente o diagnóstico da DENMO.

\section{Achados na Ressonância Magnética-Nervo Óptico}

Durante a fase aguda, a neurite óptica é comumente vista na ressonância magnética como um nervo óptico espessado com hiperintensidade em imagens ponderadas em T2 e realce em imagens ponderadas em T1 com gadolínio. ${ }^{14,18}$ Caracteriza-se pelo envolvimento do nervo óptico longitudinalmente extenso, geralmente afetando mais da metade do comprimento do nervo óptico.
Inclui tipicamente o segmento intracraniano dos nervos ópticos, geralmente estendendo-se ao quiasma e ao trato óptico (Figuras 1 e 2). ${ }^{14,19}$

A lesão medular longitudinalmente extensa observada à ressonância magnética ponderada em T2 é a lesão medular mais comum. É caracterizada pelo envolvimento longitudinal da medula espinhal em três ou mais segmentos vertebrais contíguos. ${ }^{14,18}$ A substância cinzenta central ao longo do canal central da medula espinhal é a área mais envolvida, correspondendo à expressão do antígeno AQP4. ${ }^{14}$

Amielite transversa longitudinalmente extensa é raramente observada na esclerose múltipla. A medula espinhal cervical é o segmento mais comumente afetado nesta doença. ${ }^{14} \mathrm{~A}$ mielite na esclerose múltipla, em geral, tem menor extensão longitudinal e uma distribuição mais periférica na medula espinhal, isto é, com envolvimento da substância branca.

A prevalência de lesões cerebrais na ressonância magnética pode variar de 24\%89\% nos pacientes com NMOSD. ${ }^{19,20}$ Apesar do aumento da detecção do envolvimento cerebral em pacientes com NMOSD, a maioria dessas lesões permanece inespecífica. ${ }^{20}$ Além disso, cerca de $16 \%$ desses pacientes, também satisfaz os critérios de ressonância magnética para esclerose múltipla..$^{9,14,20}$

\section{Detecção de AQP4-IgG}

Os pacientes com suspeita de NMO devem ser testados para anticorpos séricos AQP4-IgG idealmente durante os surtos da doença e preferencialmente antes da terapia imunossupressora. Pacientes que inicialmente são soronegativos para o anticorpo AQP4, mas com suspeita mantida para NMO, devem ser submetidos ao teste posteriormente, pois a conversão para o estado soronegativo pode ocorrer com a imunossupressão. ${ }^{21}$

Estudos atuais relatam moderada sensibilidade e alta especificidade do anticorpo anti-AQP4 para $\mathrm{NMO}$, com vários ensaios usados para detectar o biomarcador sérico específico. O teste recomendado pelo Painel Internacional 
Figura 1. Imagem de ressonância magnética em corte sagital pondera da em T 1 comgdacometendo medula no segmento compreendido entre $\mathrm{C} 2$ e T11. Realce anômalo pelo meio de contraste de T1 a T9 em uma paciente com diagnóstico de lúpus e artrite reumatoide (síndrome mista do tecido conjuntivo ) e AQ4 positiva.

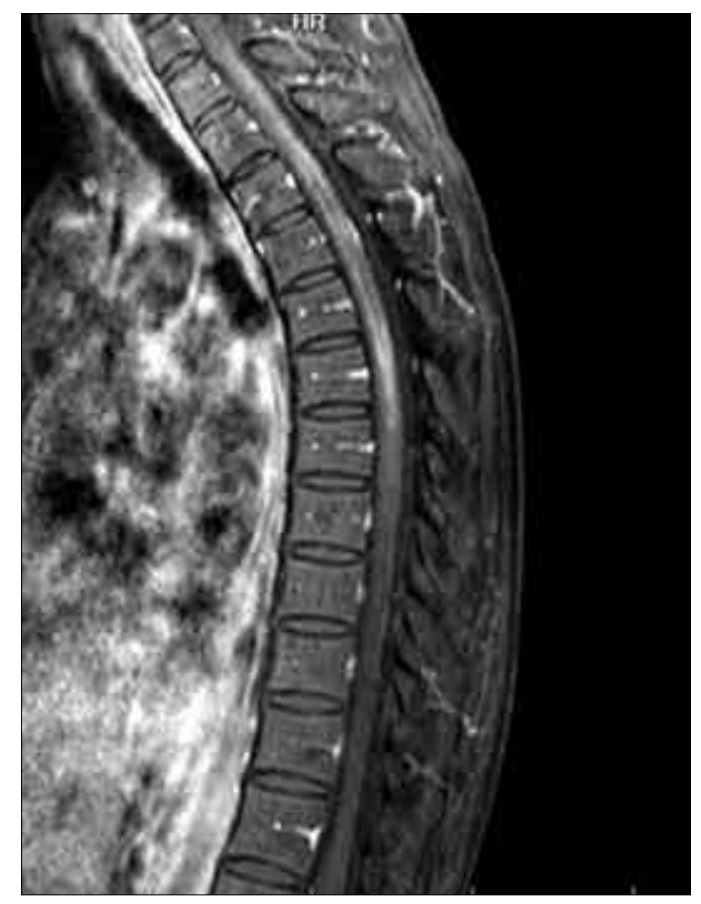

Figura 2. Imagem de ressonância magnética em corte axial ponderada em T1 com captação de contraste em nervo óptico esquerdo de paciente jovem masculino com diagnóstico de DENMO.

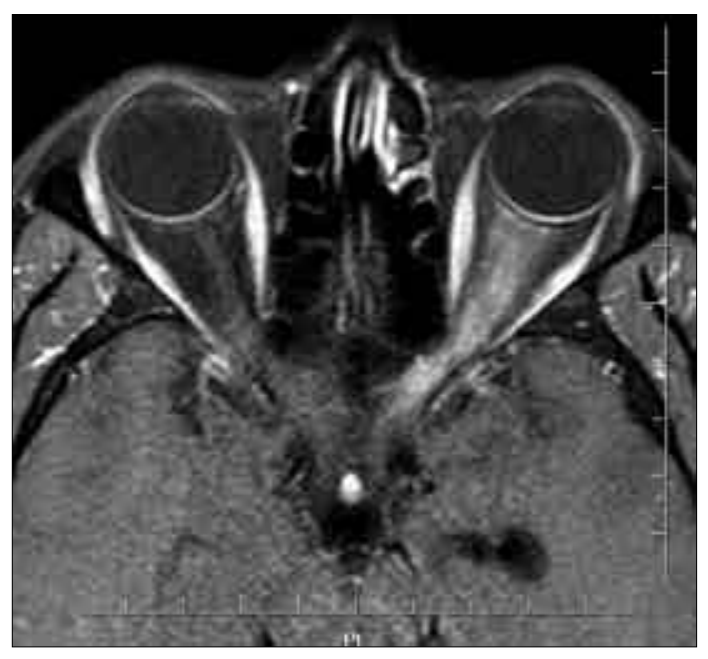

para Diagnóstico NMO é o ensaio baseado em células-CBA, que possui sensibilidade média e especificidade de $76,7 \%$ e $99,8 \%$, respectivamente. ${ }^{14}$

No entanto, uma proporção de pacientes (20\%-40\%), a depender da coorte estudada, com um fenótipo típico de $\mathrm{NMO}$, é negativa para anticorpos AQP4, mesmo com a utilização de testes mais sensíveis. Dados limitados sugerem que a DENMO seronegativa pode diferir da NMOSD soropositiva em certas características. ${ }^{22}$

A razão sexual entre homens e mulheres na NMO positivo para anticorpo-MOG é de aproximadamente 1 , comparada com 0,1 ou 0,2 em pacientes com anticorpo AQP4. Os pacientes positivos para anticorpo-MOG apresentam mais frequentemente neurite óptica isolada ou mielite e doença monofásica, mesmo após longo período de acompanhamento. Os sintomas em pacientes positivos para anticorpo-MOG tendem a ser menos graves com recuperação e resposta a corticosteroides melhores do que em pacientes com anticorpos para AQP4. A ressonância magnética em pacientes positivos para anticorpoMOG revelou lesões cerebrais e toracolombares mais frequentes no curso da doença, quando comparados com pacientes NMOSD positivos para anticorpos AQP4, nos quais as lesões foram localizadas na medula espinhal cervico torácica e tronco cerebral. ${ }^{22}$

O papel dos anticorpos séricos da imunoglobulina contra a $\mathrm{MOG}$ em pacientes com desmielinização do SNC permanece em aberto, sendo possível que eles sejam expressos secundariamente ao dano tecidual. Vale salientar ainda que os critérios revisados de 2015 para DENMO relacionados aos anticorpos AQP4 ainda não são aplicáveis aos anticorpos MOG. ${ }^{22}$

$\mathrm{O}$ conhecimento sobre a especificidade dos anticorpos MOG, mesmo com ensaios baseados em células, ainda é limitado. Outra questão seria a valorização dos anticorpos contra MOG em populações pediátricas. Os anticorpos MOG são encontrados em numerosas síndromes desmielinizantes em crianças e podem ser transitórios. A repetição dos testes é obrigatória 
Tabela 1. Critérios diagnósticos para DENMO em pacientes adultos. ${ }^{12}$

\section{Critérios para Diagnóstico de DENMO com AQP4-IgG}

1. Pelo menos 1 dos sintomas centrais no quadro clínico;

2. Dosagem positiva para AQP4-IgG. Presença de IgGanti AQP4;

3. Exclusão de outros diagnósticos.

\section{Critérios para Diagnóstico de DENMO sem AQP4-IgG ou DENMO com Status Desconhecido de AQP4-IgG}

1. Ao menos 2 características clínicas centrais resultado de um ou mais crises agudas e todos os seguintes requisitos:

a. Ao menos 1 das características clínicas centrais devem ser neurite óptica, mielite aguda com METL ou síndrome da área postrema;

b. Disseminação no espaço ( 2 ou mais características clínicas diferentes).

c. Preenche critérios na RNM se possível;

2. Ausência de anticorpo anti AQP4-IgG ou teste indisponível;

3. Exclusão de outros diagnósticos.

\section{Características Clínicas Centrais}

1. Neurite óptica;

2. Mielite aguda;

3. Síndrome da Área Postrema: episódios de soluços ou náuseas/vômitos sem outras explicações;

4. Síndrome do tronco cerebral aguda;

5. Narcolepsia sintomática ou síndrome diencefálica aguda com RNM de crânio característica de DENMO;

6. Síndrome cortical sintomática com lesões encefálicas típicas de DENMO.

\section{Achados na RNM Necessários para DENMO sem AQP4-IgG e DENMO com Status Desconhecido} de AQP4-IgG

1. Neurite Óptica Aguda: RNM de crânio mostrando (a) achados normais ou apenas lesões inespecíficas da substância branca, OU (b) RNM com nervo óptico com lesões hiperintensas em T2 ou lesão que é contrastada em T1 abrangendo $>1 / 2$ da extensão do nervo óptico ou envolvendo o quiasma óptico;

2. Mielite Aguda: RNM com Mielite transversa longitudinalmente extensa - MTLE (lesões intramedulares estendendo por $>3$ segmentos contínuos) OU $>3$ segmentos contínuos com atrofia focal da medula espinal em pacientes com história compatível com mielite aguda;

3. Síndrome da área postrema: requer lesões associadas da medula dorsal/área postrema;

4. Síndrome do tronco encefálico: requer lesões associadas na região periependimal do tronco encefálico.

AQP4 = aquaporina-4; IgG = imunoglobulina G; MTLE = mielite transversa longitudinalmente extensa; NMOSD = doenças do espectro neuromielite óptica. Adaptado da International Consensus Diagnostic Criteria for Neuromyelitis Optica Spectrum Disorders. ${ }^{12}$

antes de um diagnóstico definitivo de uma doença crônica por anticorpos MOG. ${ }^{22}$

\section{Líquido Cefalorraquidiano}

As anormalidades dolíquido cefalorraquidiano são detectadas na maioria dos pacientes com NMO e estão relacionadas à contagem de células, proteína e bandas oligoclonais. A análise do liquido cefalorraquidiano deve ser obtida durante ou logo após um ataque agudo. ${ }^{14,23}$

A pleocitose, geralmente constituída por monócitos e linfócitos, está presente em 14\%$79 \%$ dos pacientes, podendo incluir ou ter predominância neutrofílica. A contagem de 
células do liquido cefalorraquidiano é maior que 50 células / $\mu \mathrm{L}$ em 13\%-35\% dos pacientes e, em alguns casos, até 1.000 células / $\mu \mathrm{L}$. $^{23}$

Pacientes com LETM são mais propensos a apresentarpleocitose do que pacientes comneurite óptica. Os níveis de proteína aumentados estão presentes em $46 \%-75 \%$ dos casos. A frequência de bandas oligoclonais em NMO varia de $0 \%$ a $37 \%$ e pode ser transitória. A análise do líquido cefalorraquidiano na EM raramente revela uma pleocitose superior a 50 células / $\mu \mathrm{L}$, com bandas oligoclonais presentes em mais de $90 \%$ na EM estabelecida. ${ }^{23}$

Assim, a análise do LCR fornece dados de suporte para o diagnóstico de NMO e achados no LCR com pleocitose linfomononuclear $>50$ células/ $\mu \mathrm{L}$, presença ocasional de neutrófilos/ eosinófilos e ausência de bandas oligoclonais podem ser indicativos, mas não específicos, de DENMO e NMO. ${ }^{23}$

\section{Diagnóstico Diferencial}

O principal diagnóstico diferencial é a esclerose múltipla, distúrbio desmielinizante mais comum do SNC, além da encefalomielite disseminada aguda. Lesões medulares extensas podem se apresentar em outras condições, tais quais lúpus eritematoso sistêmico, Sjögren, sarcoidose, esclerose múltipla, causas vasculares e metabólicas, além de infecções pelo vírus da imunodeficiência humana. ${ }^{14,18}$

\section{Preditores de Pior Prognóstico}

Preditores de pior prognóstico incluem o número de recaídas nos primeiros dois anos da doença, gravidade do primeiro ataque e idade mais avançada no início da doença. ${ }^{18} \mathrm{~A}$ maioria das recidivas ocorre por vários dias e melhora nas semanas ou meses subsequentes após o máximo comprometimento clínico. A recuperação é geralmente incompleta e a maioria os pacientes segue um curso de incapacidade incremental devido a recidivas frequentes e graves. ${ }^{5}$

\section{Tratamento}

O tratamento da DENMO pode ser dividido em tratamento das exacerbações agudas (surtos) e o tratamento modificador da doença. O tratamento das exacerbações agudas tem como objetivo reduzir a intensidade, o tempo de resolução e as sequelas dos pacientes. O tratamento modificador de doença tem por objetivo reduzir a frequência e intensidade dos surtos.

A pulsoterapia com metilprednisolona por via venosa por 3 a 5 dias é comumente o tratamento inicial para ataques agudos da NMO (recomendação do painel de especialistas e baseada em estudos de esclerose múltipla e neurite óptica idiopática). ${ }^{21,22}$ Pacientes que não respondem ao tratamento com corticosteroides beneficiam-se de plasmaférese como tratamento de resgate. ${ }^{21,22}$

No último ano, avanços significativos vêm ocorrendo no tratamento modificador da doença, ainda não incorporados à prática clínica. Em décadas passadas, a maioria dos pacientes era diagnosticada com esclerose múltipla grave e tratada com terapias tradicionais para tratamento de esclerose múltipla. As terapias avaliadas para DENMO como interferon-beta, natalizumabe, fingolimode e alentuzumabe não foram benéficas e apresentaram risco de piorar o curso clínico dos pacientes com DENMO. ${ }^{23,24}$

Éimportanteressaltarqueaterapia demanutenção praticada atualmente é baseada apenas em estudos observacionais, estudos clínicos de baixa qualidade metodológica e recomendações de consensos elaboradas por especialistas. No momento, não existe nenhuma droga aprovada pelas agências regulatórias no Brasil, Estados Unidos ou Europa para a doença. Com base em recomendações de sociedades como o Comitê Brasileiro de Tratamento e Pesquisa em Esclerose Múltipla (BACTRIMS), as medicações consideradas como primeira linha terapêutica são a azatioprina ou azatioprina + prednisona, o rituximabe e o micofenolatomofetila, e como segunda linha medicações menos estudadas para DENMO como o metrotexate e a ciclofosfamida. ${ }^{25}$ 
Uma coorte observacional realizada no Brazil, naUniversidadeFederaldeSãoPaulo, demonstrou que a azatioprina (2-3mg/kg/dia) combinada com prednisona oral $(1 \mathrm{mg} / \mathrm{kg} / \mathrm{dia})$ reduz $\mathrm{o}$ número de crises efetivamente. ${ }^{26} \mathrm{O}$ rituximabe é um anticorpo monoclonal quimérico capaz de bloquear o receptor de superfície CD20, presente nas células precursoras do linfócito $B$, que é um inibidor de CD20. Estudos iniciais demonstraram uma redução da frequência anualizada de surtos em paciente em uso do rituximabe.

O estudo inicial com rituximabe na NMO foi realizado por Cree e colaboradores, ${ }^{27}$ usando $375 \mathrm{mg} / \mathrm{m}^{2}$ de superfície corporal, uma vez por semana, por quatro semanas como indução, e novas aplicações conforme monitoramento da porcentagem de células CD20, bimensalmente ("protocolo Cree"). Atualmente, tem-se utilizado protocolos de indução com dose de 1 grama repetido após 15 dias, mantendo-se 1-2 gramas semestralmente. Estudo comparando a taxa anual de surto da azatioprina, do micofenolato de mofetila e do rituximabe evidenciou superioridade do último em relação à azatioprina e eficácia similar àquela do micofenolato de mofetila. Outro estudo comparativo entre estas medicações confirma esses achados edemonstrou que o rituximabe é superior à azatioprina e ao micofenolato de mofetila para controle dos surtos graves. Em caso de resposta terapêutica insatisfatória ou efeitos colaterais relevantes, a realização de troca entre essas medicações pode ser indicada. ${ }^{28}$

O melhor entendimento fisiopatológico e a descoberta de novos alvos terapêuticos têm proporcionado o desenvolvimento de drogas promissoras para o tratamento de manutenção da DENMO. O bloqueio de interleucinas associadas com ativação das células Th17 particularmente anti-receptor de IL6 tem se mostrado promissor na terapia de manutenção de pacientes tanto associado com outras terapias como em monoterapia. Um estudo randomizado realizado utilizando o satrilizumabe demonstrou uma redução de $62 \%$ das exacerbações clínicas quando comparado ao placebo. ${ }^{28}$ Outro alvo terapêutico promissor é o uso de inibidores de complemento.Umestudopublicadorecentemente na revista New England Journal of Medicine $(2019)^{29}$ demonstrou que o eculizumabe frente ao placebo reduziu em mais de $94 \%$, no período de 48 semanas estudadas, as exacerbações clínicas da doença. Outra promessa são os inibidores de CD19. Um trabalho apresentado no Congresso da Academia Americana de Neurologia, em maio de 2019, com a medicação inebilizumab, demonstrou uma redução de $77 \%$ dos eventos em pacientes tratados. ${ }^{30}$

Nós últimos 120 anos, desde o a descrição clínica da doença, todos os tratamentos realizados para tratamento da DENMO foram baseados em evidência de baixa qualidade. No entanto, é provável que neste último ano e nos próximos que virão as evidências produzidas mudarão nosso entendimento sobre a doença e, radicalmente, a forma dos tratamentos.

\section{Referências}

1. Bennett JL. Finding NMO: the evolving diagnostic criteria of neuromyelitis optica. J Neuroophthalmol. 2016;36(3):238-45.

2. Flanagan EP, Cabre $P$, Weinshenker BG, et al. Epidemiology of aquaporin-4 autoimmunity and neuromyelitis optica spectrum. Ann Neurol. 2016;79(5):775-83.

3. Lennon VA, Wingerchuk DM, Kryzer TJ, et al. A serum autoantibody marker of neuromyelitis optica: distinction from multiple sclerosis. Lancet 2004;364(9451):210612.

4. Roemer SF, Parisi JE, Lennon VA, et al. Pattern-specifi c loss of aquaporin-4 immunoreactivity distinguishes neuromyelitis optica from multiple sclerosis. Brain 2007;130:1194-205.

5. Water s PJ, McKeon A, Leite MI, et al. Serologic diagnosis of NMO: a multicenter comparison of aquaporin-4-IgG assays. Neurology 2012;78:665-71.

6. Mealy MA, Wingerchuk DM, Greenberg BM, Levy M. Epidemiologia da neuromielite óptica nos Estados Unidos: uma análise multicêntrica. Arch Neurol. 2012;69:1176.

7. Papadopoulos MC, Verkman AS. Aquaporin 4 and neuromyelitis optica. Lancet Neurol. 2012;11:535.

8. Sellner J, Boggild M, Clanet M, et al. EFNS guidelines on diagnosis and management of neuromyelitis optica. Eur J Neurol. 2010;17:1019. 
9. Kim SH, Kim W, Li XF, et al. Clinical spectrum of CNS aquaporin-4 autoimmunity. Neurology 2012;78:1179.

10. Matiello M, Kim HJ, Kim W, et al. Familial neuromyelitis optica. Neurology 2010; 5:310.

11. Pittock SJ, Lucchinetti CF. Neuromyelitis optica and the evolving spectrum of autoimmune aquaporin-4 channelopathies: a decade later. Ann N Y Acad Sci. 2016;1366(1):20-39.

12. Zhang B, Zhong Y, Wang Y, et al. Neuromyelitis optica spectrum disorders without and with autoimmune diseases. BMC Neurol. 2014;14(1):162.

13. Titulaer MJ, Höftberger R, Iizuka T, et al. Overlapping demyelinating syndromes and anti-N-methylD-aspartate receptor encephalitis. Ann Neurol 2014;75(3):411-28.

14. Dutra BG, Rocha AJ, Nunes RH, et al. Neuromyelitis optica spectrum disorders: spectrum of MR imaging findings and their differential diagnosis. Neurologic/ Head. 2018;38:169-93.

15. Kitley J, Woodhall M, Waters P, et al. Myelinoligodendrocyte glycoprotein antibodies in adults with a neuromyelitis optica phenotype. Neurology 2012;79:1273.

16. Sato DK, Callegaro D, Lana-Peixoto MA, et al. Distinction between MOG antibody-positive and AQP4 antibody-positive NMO spectrum disorders. Neurology 2014;82:474.

17. Wingerchuk DM, Hogancamp WF, O'Brien PC, Weinshenker BG. The clinical course of neuromyelitis optica (Devic's syndrome). Neurology 1999; 3:1107.

18. Wingerchuk DM, Lennon VA, Lucchinetti CF, Pittock SJ, Weinshenker BG. The spectrum of neuromyelitis optica. Lancet Neurol. 2007;6(9):805-15.

19. Ramanathan S, Prelog K, Barnes EH, et al. Radiological differentiation of optic neuritis with myelin oligodendrocyte glycoprotein antibodies, aquaporin- 4 antibodies, and multiple sclerosis. Mult Scler. 2016;22(4):470-82.

20. Wang F, Liu Y, Duan Y, Li K. Brain MRI abnormalities in neuromyelitis optica. Eur J Radiol. 2011;80(2):445-9.

21. Sellner J, Boggild M, Clanet M, et al. EFNS guidelines on diagnosis and management of neuromyelitis optica. Eur J Neurol. 2010;17:1019.
22. Trebst C, Jarius S, Berthele A, et al. Update on the diagnosis and treatment of neuromyelitis optica: recommendations of the Neuromyelitis Optica Study Group (NEMOS). J Neurol. 2014; 261:1.

23. Kim SH, Kim W, Li XF, Jung IJ, Kim HJ. Does interferon beta treatment exacerbate neuromyelitis optica spectrum disorder? Mult Scler. 2012 Oct;18(10):1480-3.

24. Kleiter I1, Hellwig K, Berthele A, Kümpfel T, Linker RA, Hartung HP, Paul F, Aktas O; Neuromyelitis Optica Study Group. Failure of natalizumab to prevent relapses in neuromyelitis optica. Arch Neurol. 2012 Feb;69(2):239-45.

25. Frota E. et al.. Recomendações no Tratamento da Esclerose Múltipla e Neuromielite Óptica. 2. ed. São Paulo: Editora e Eventos Omnifarma, 2016.

26. Bichuetti DB, Lobato de Oliveira EM, Oliveira DM, Amorin de Souza N, Gabbai AA. Neuromyelitis optica treatment: analysis of 36 patients. Arch Neurol. 2010;67(9):1131-6.

27. Mealy MA, Wingerchuk DM, Palace J, Greenberg BM, Levy M. Comparison of relapse and treatment failure rates among patients with neuromyelitis optica: multicenter study of treatment efficacy. JAMA Neurol. 2014;71(3):324-30.

28. Sean J. Pittock, M.D., Achim Berthele, MD, Kazuo Fujihara. Eculizumab in Aquaporin-4-Positive Neuromyelitis Optica Spectrum Disorder. N Engl J Med. 2019 May 3.

29. Yamamura T, Kleiter I, Fujihara K, et al. Efficacy of satralizumab (SA237) in subgroups of patients in SAkuraSky: a Phase III double-blind, placebocontrolled, add-on study in patients with neuromyelitis optica spectrum disorder (NMOSD). American Academy Neurology Congress Abstracts 2019S43.008.

30. Cree B, Bennett J, Kim, Ho J, Weinshenker B, Pittock S. A double-masked, placebo-controlled study with open-label period to evaluate the efficacy and safety of inebilizumab in adult subjects with neuromyelitis optica spectrum disorders - top line efficacy and safety results. American Academy Neurology Congress Abstracts, 2019;Plen02.001. 